\title{
PHARMACEUTICAL AMORPHOUS ORGANIC MATERIALS CHARACTERIZATION BY USING THE DIFFERENTIAL SCANNING CALORIMETRY AND DYNAMIC MECHANICAL ANALYSIS
}

\author{
Ion Dranca, ${ }^{*}$ Igor Povar and Tudor Lupascu \\ Institute of Chemistry, Academy of Sciencesof Moldova \\ 3 Academiei Str., Chisinau MD 2028, Republic of Moldova.e-mail: drancai@yahoo.com
}

\begin{abstract}
This research has been carried out in order to demonstrate the use of differential scanning calorimetry (DSC) in detecting and measuring $\alpha$ - and $\beta$-relaxation processes in amorphous pharmaceutical systems. DSC has been employed to study amorphous samples of poly (vinylpyrrolidone) (PVP), indomethacin (InM), and ursodeoxycholic acid (UDA) that are annealed at temperature $\left(T_{a}\right)$ around 0.8 of their glass transition temperature $\left(T_{q}\right)$. Dynamic mechanical analysis $(D M A)$ is used to measure $\beta$ - relaxation in PVP. Yet, the DSC has been used to study the glassy indomethacin aged at 0 and $-10^{\circ} \mathrm{C}$ for periods of time up to 109 and 210 days respectively. The results demonstrate the emergence of a small melting peak of the $\alpha$-polymorph after aging for 69 days at $0^{\circ} \mathrm{C}$ and for 147 days at $-10^{\circ} \mathrm{C}$ (i.e., $\sim 55^{\circ} \mathrm{C}$ below the glass transition temperature) that provides evidence of nucleation occurring in the temperature region of the $\beta$-relaxation. The evolution of an endothermic recovery peak temperature features a plateau at longer annealing times, suggesting that the glass has made a significant progress toward reaching the supercooled liquid state. It has been found that the melting peaks become detectable after the recovery peak reaches the plateau. The results highlight the importance of studying physical aging in the temperature region of the $\beta$ - relaxation as a means of evaluating the physical stability of amorphous pharmaceutical materials.
\end{abstract}

Keywords: DSC - differential scanning calorimetry; DMA - dynamic mechanical analysis; drugs; excipients; glass transition; $\alpha$ - and $\beta$-relaxations; crystallization; kinetics.

\section{Introduction}

Since amorphous compounds are inherently less stable, both physically and chemically, than their crystalline counterparts, numerous investigations over the last decade expectedly focused on the stabilization of amorphous pharmaceuticals [1]. DSC has been used to probe the $\beta$-relaxation for a large variety of polymers [2]. Although dielectric spectroscopy has been applied to the relaxation of indomethacin, the studies have been focused on the temperature region of the $\alpha$-relaxation, and the $\beta$-process has not been reported.

\section{General application of DTA and DSC}

The results obtained using DTA and DSC are qualitatively so similar that their application usually is not treated separately. It should be noted that DTA can be used to higher temperatures than DSC $\left(\max 725^{\circ} \mathrm{C}\right)$ but that more reliable quantitative information obtained from DSC [3]. Differential scanning calorimetry (DSC) is the most frequently used thermal analysis technique alongside TGA, TMA and DMA. DSC is used to measure enthalpy changes (figure 1) due to changes in the physical and chemical properties of a material as a function of temperature or time. The method allows identifying and characterizing materials including pharmaceuticals. DSC is the method of choice to determine thermal quantities, study thermal processes, and characterize or just simply compare materials. The Dynamic Mechanical Analysis (DMA) basic principles [3] can be simply described as applying an oscillating force to a sample and analyzing the material's response to that force (figure 2). From DMA, one calculates properties like tendency to flow (called viscosity) from the phase lag and the stiffness (modulus) from the sample recovery.

These properties are often described as the ability to lose energy as heat (damping) and the ability to recover from deformation (elasticity). The applied force is called stress and is denoted by the Greek letter, $\sigma$. When subjected to stress, a material will exhibit a deformation or strain, $\gamma$. The sample is subjected to a periodically varying stress (usually sinusoidal of angular frequency, $\omega$ ). The response of the sample to this treatment can provide information on the stiffness of the material (quantified by its elastic module) and its ability to dissipate energy (measured by its damping). For a viscoelastic material, the strain resulting from the periodic stress is also periodic, but it is out of phase with the applied stress owing to energy dispersion as heat, or damping in the sample [3]. A brief history of the DMA technique is given in the book by Menard [5]. 


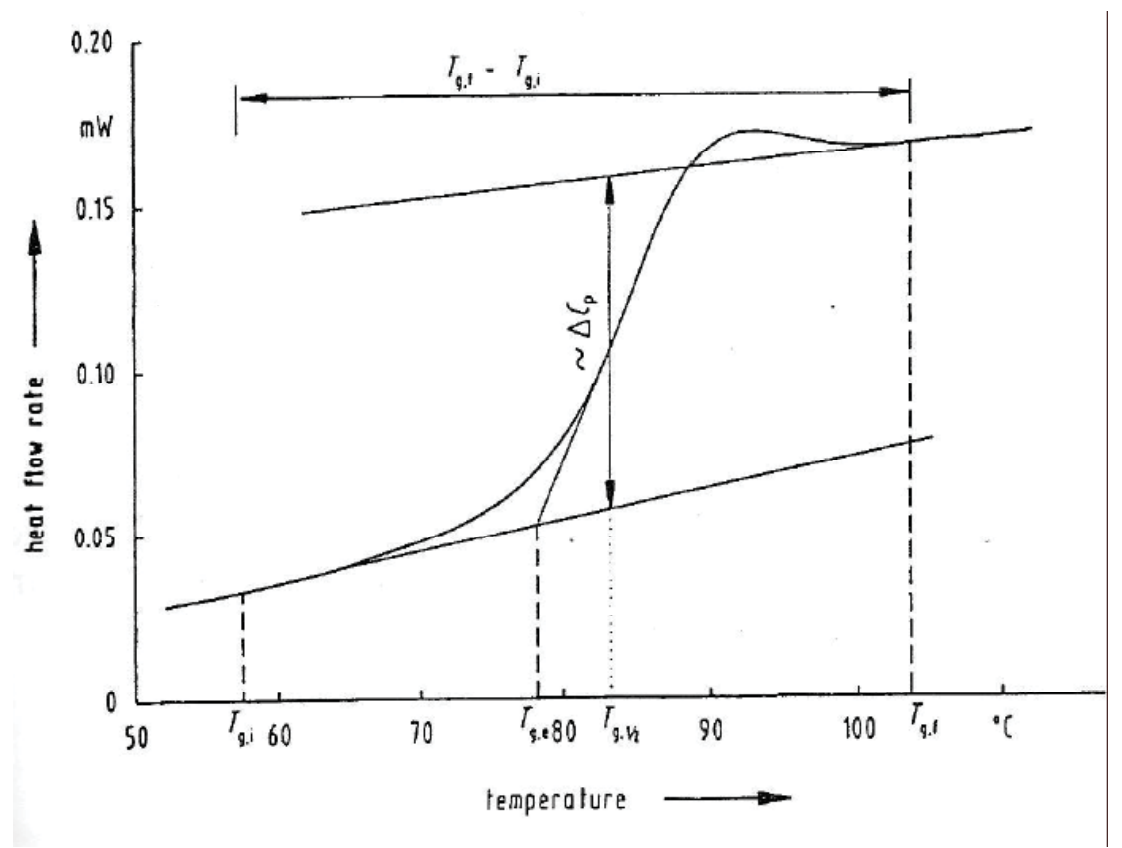

Figure 1. Definition of characteristic features of the glass transition. $T_{g . e}=$ the extrapolated onset temperature; $T_{g}$, $=$ the half-step temperature at which the heat capacity is midway between the extrapolated heat capacities of the liquid and glassy states; $\mathrm{T}_{\mathrm{g} . \mathrm{i}}$ and $\mathrm{T}_{\mathrm{g} . \mathrm{f}}$ are the initial and final temperatures of the glass transition and $\mathrm{T}_{\mathrm{g} . \mathrm{f}}-\mathrm{T}_{\mathrm{g} . \mathrm{i}}$ is the temperature interval of the glass transition [4]

(a)

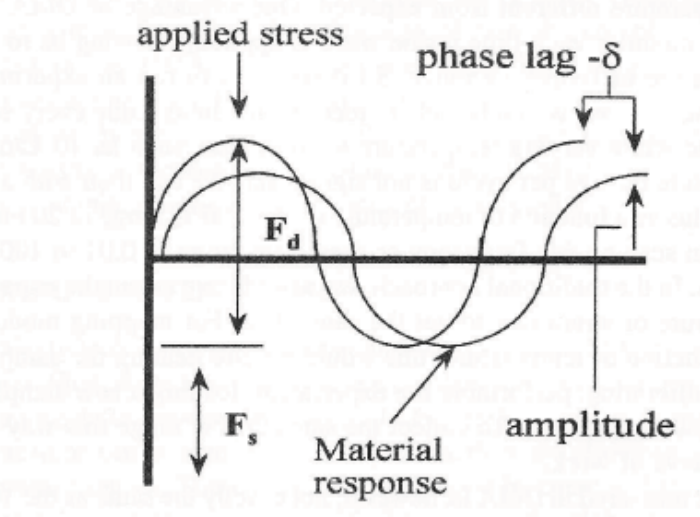

(b)

Figure 2. How DMA works. The DMA supplies an oscillatory force causing a sinusoidal stress to be applied to the sample, which generates a sinusoidal strain. By measuring both the amplitude of deformation at the peak of the sine wave and lag between the stress and strain waves, quantities like the modulus, the viscosity, and the dumping can be calculated

\section{General Mechanism of the $\beta$-transition in Polymers and Pharmaceutics}

V. A. Bershtein and V. M. Egorov have been pointed [6], in 1985 that the result of DSC investigations shows that in polymers relaxation processes the $\beta$-transition is a general phenomenon common to no crystallizing solids. A relationship has been found between thermo activation parameters of the transition and molecular characteristics i. e. the average length of a molecule, the length of the statistical Kuhn segment, the cohesive energy and the potential barrier of internal rotation. It is concluded that the act of $\beta$ - transition is comparable to acts of relaxation in liquids, and take place in sites of less dense packing. For linear nonrigid polymers the act consist in the rotation of a chain fragment similar in length to the Kuhn segment involving the surmounting of preferentially intermolecular barriers with the participation of $\mathrm{S}-\mathrm{G}$ transition; the kinetic unit corresponding to the $\beta$-transition is related to the segment of intermolecular mobility in polymers in pharmaceutics.

The use of the amorphous form of drugs and excipients to improve solubility, accelerate dissolution and promote 
therapeutic activity has been advocated by many workers [7]. The rationale behind such a strategy is that a highly disordered amorphous material has a lower energetic barrier to overcome in order to enter solution than a regularly structured crystalline solid. In order to produce a usable amorphous system it is necessary to create a highly disordered molecular state (usually by a high energy process such as milling or lyophilisation) and then to stabilize that disordered state (usually by rapid drying or cooling or addition of stabilizing agents) so that all molecular motions which might induce instability are retarded over a meaningful pharmaceutical time-scale. It is the apparent chemical and physical instability of most amorphous pharmaceutical solids which is the major precluding their more widespread use in solid dosage forms [8].

Amorphous pharmaceuticals draw a great deal of interest because of their potential to enhance bioavailability. But downside of amorphous materials is their thermodynamic instability that unavoidable drives them toward the stable crystalline state.

Excipients are secondary ingredients added to a formulation to aid the delivery, processing and stability of the active drug/ drugs. Formulation stability largely depends on interaction of excipients or lack of interaction of excipients with the active drug and other excipients. Excipients are the additives used to convert pharmacologically active compounds into pharmaceutical dosage form suitable for administration to patients. Careful selection of excipients is critical for maintaining the physical structure of the dosage form, prevention of degradation of the active drug and achieving the desired bioavailability [9]. DSC has been a widely used method to analyze drug- excipient compatibility. Compared to the traditional compatibility methods DSC offers significant advantages by saving time and utilizing smaller amounts of sample. Significant changes in the DSC profile of a mixture compared to those of the individual components indicate probable interactions. Changes in the melting point of the components, peak area, shape, broadening or elongation of the endothermic or exothermic peaks, appearance of new peaks are the indicators of interactions $[10,11]$.

\section{Experimental section}

PVP (MW 8,000), indomethacin and ursodeoxycholic acid were used from Fisher. All the substances were used without further purification. For the annealing ( $\beta$-relaxation) measurements, about $20 \mathrm{mg}$ of crystalline InM or UDA was placed in $40 \mu \mathrm{L} \mathrm{Al}$ pans and heated to $\sim 15^{\circ} \mathrm{C}$ above their respective melting points, 160 and $205^{\circ} \mathrm{C}$. In the case of PVP, the samples were about $12 \mathrm{mg}$ and heating was conducted up to $\sim 70^{\circ} \mathrm{C}$ above its glass transition temperature of $140^{\circ} \mathrm{C}$. Immediately after heating, the samples were quenched into liquid nitrogen and quickly placed into the DSC (Mettler-Toledo DSC $822^{\circ}$ ) that was maintained at $20^{\circ} \mathrm{C}$ for PVP and UDA and at $-40^{\circ} \mathrm{C}$ for InM runs. After a short period of stabilization at the initial temperature, the samples were heated to an annealing temperature, $T_{a}$, and maintained for $30 \mathrm{~min}$. The annealing temperatures were $-20,-10$, and $0^{\circ} \mathrm{C}$ for $\mathrm{InM} ; 50,60,7080$, and $90^{\circ} \mathrm{C}$ for PVP; and for $30,40,50$, and $60^{\circ} \mathrm{C}$ for UDA. After completion of the annealing segment, the samples were cooled down either to $-10^{\circ} \mathrm{C}$ (PVP and UDA) or to $-40^{\circ} \mathrm{C}$ (InM), and immediately heated above $T_{g}$. The heating rates were 10 , $15,20,25$, and $30^{\circ} \mathrm{C} \mathrm{min}^{-1}$.

\section{Results and discussion}

In this paper we demonstrate, that $\beta$-relaxation in pharmaceutical glasses can be probed via DSC and DMA

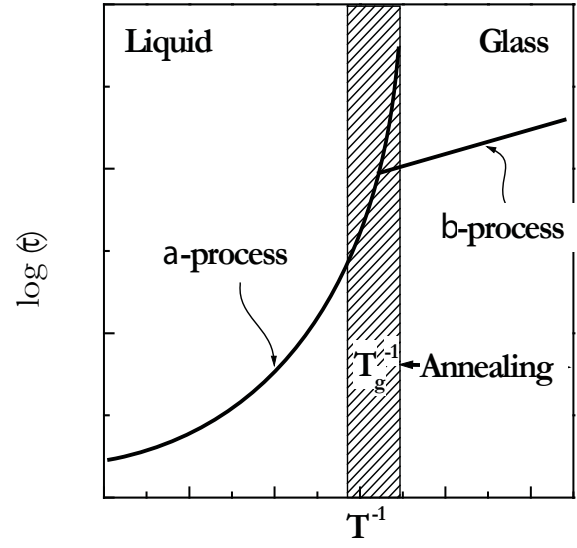

Fig. 3. Temperature dependence of relaxation time for the $\alpha$ - and $\beta$-processes. Hatched area represents the region around $T_{g}$, where the $\alpha$ - and $\beta$-processes are strongly coupled

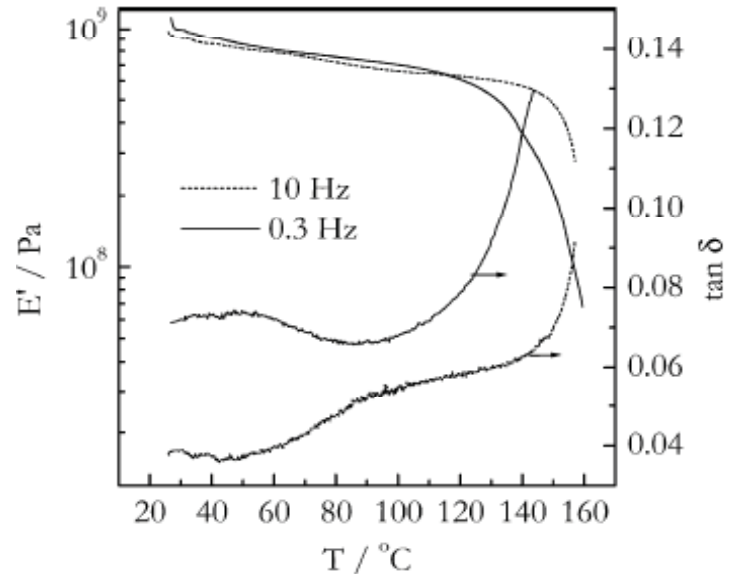

Fig. 4. DMA data (storage modulus, $E^{\prime}$, and loss tangent, $\tan \delta$ ) for PVP sample at the two extreme frequencies 
measurements of the aforementioned annealing effects. We have applied this method to several pharmaceutically relevant glasses that include poly(vinylpirrolidone) (PVP), indomethacin (InM), and ursodeoxycholic acid (UDA) and demonstrate the $E$ values obtained as:

$$
E=-R \frac{d \ln q}{d T_{p}^{-1}}
$$

and that the activation energy, $E$, evaluated from the shift of the peak temperature, $T_{p}$, with the heating rate, $q$, as (1). The consistency is confirmed by comparing the obtained $E$ values with independent measurements, literature values, and the empirical correlation, $E_{\beta}=(24 \pm 3) R T_{g}$, discovered by Kudlik et al.[12]. Yet, the importance of low temperature mobility of amorphous pharmaceuticals is well recognized [8].

Figure 5 displays variation of the effective activation energy with the average peak temperature for DSC and DMA.

\section{Nucleation of amorphous indomethacin}

Indomethacin (indometacin) (1-(p-chlorobenzoyl)-5-methoxy-2-metylindole-3-acetic acid) from MP Biomedicals, LLC was used without further purification. After quick melting samples were quenched into liquid nitrogen. The quenched samples had the value of $T_{g}=46^{\circ} \mathrm{C}$ that was determined by DSC as a midpoint of the glass transition step observed on heating at $10^{\circ} \mathrm{C} \mathrm{min}^{-1}$.

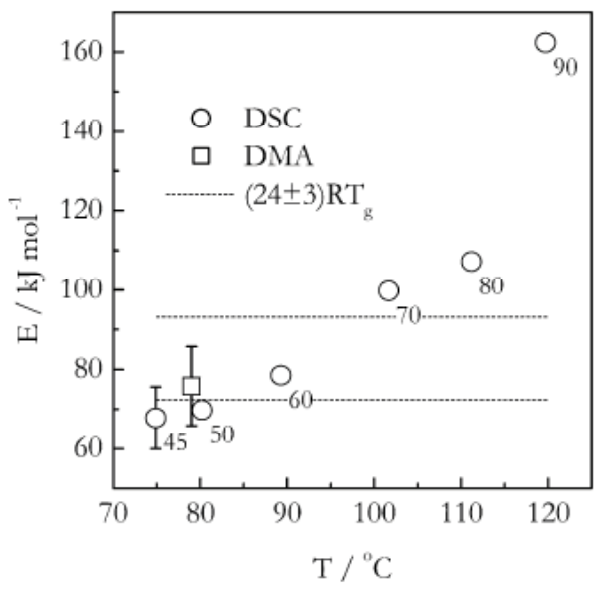

Fig. 5. Variation of the effective activation energy with the average peak temperature for DSC and DMA data on PVP. Numbers by the points represent annealing temperatures. Error bars are confidence intervals. Dash lines set lower and upper limits for $(24 \pm 3) R T_{g}$

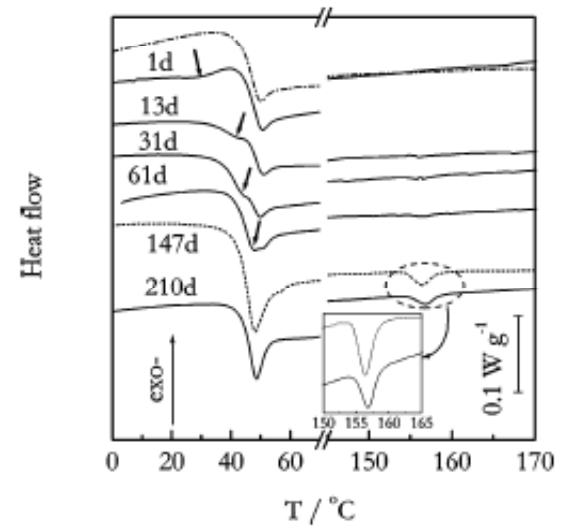

Fig. 6. DSC curves obtained on heating at $10^{\circ} \mathrm{C}$ $\mathrm{min}^{-1}$ of indomethacin after long-term annealing at $T_{a}=-10^{\circ} \mathrm{C}$. The numbers by the curves represent annealing time in days (d). Dash-dot line represents non-aged glass. Arrows show the location of the annealing peak. The melting region is circled. Inset shows a blow-up of the melting region

The quenched samples were placed in freezers kept at 0 and $-10^{\circ} \mathrm{C}$ and annealed there for an extended period of time. In samples at $T_{a}=-10^{\circ} \mathrm{C}$, no melting peaks are detected at annealing times, $t_{a}$, less than 147 days (figure 6). We believe that our data on detecting the melting peaks after prolonged aging make a convincing case that nucleation does occur during aging. Although an alternative interpretation could be that that nucleation occurs not during aging but on heating of extensively aged glasses from $T_{a}$ to $T_{m}$ we consider it very unlikely.

\section{Excipients}

The local and global mobility, in two popular amorphous pharmaceutical excipients, sucrose and trehalose, have been compared. Trehalose, having a lower free volume in the glassy state due to a more tightly packed structure, experiences a greater effect of temperature on the reduction in the activation energy barrier for the glass to start flowing. This could be important in causing collapse of a lyophilized cake in that a small increase in temperature around the glass transition temperature of a formulation containing trehalose could cause pronounced viscous flow. In spite of a large difference in $T_{g}$, sucrose and trehalose are shown to have similar size of cooperatively rearranging regions (CRR) and also approximately the same number of molecules constituting the CRR, thus implying similar dynamic heterogeneity. 


\section{Conclusions}

1. DSC can be used for detecting $\beta$-relaxation processes and estimating its low temperature limit, i.e., the temperature below which amorphous drugs would remain stable.

2. It can also provide comparative estimates of low temperature stability of amorphous drugs in terms of the activation energies of the $\beta$-relaxation.

3. Physical aging of indomethacin has been studied at temperatures down to $T_{g}-56^{\circ} \mathrm{C}$ or $0.83 T_{g}$ that corresponds to the temperature region of the $\beta$-relaxation. The study demonstrates that indomethacin undergoes nucleation in this temperature region. To our knowledge, this represents the lowest temperatures, for which evidence of nucleation has been reported. Although the process is quite slow, detecting it is very important because pre-nucleated amorphous pharmaceuticals would crystallize faster when the storage temperature is increased. Clearly, studies of the kinetics of the sub- $T_{g}$ relaxations should play a key role in estimating the physical stability of amorphous pharmaceuticals. Another finding of this work is that nucleation becomes detectable after glassy indomethacin has reached a significant extent of relaxation defined as a plateau in the plot of the aging peak temperature against aging time. The significance of this correlation is that it could be used for estimating the life-times of amorphous pharmaceuticals as the time of reaching the aforementioned plateau, because this period appears to determine the time to nucleation. However, whether this correlation is a common feature of crystallizable glasses remains to be seen in our ongoing investigation.

4. DSC has been employed for probing $\beta$-relaxations in pharmaceutical glasses annealed at temperatures around $0.8 T_{g}$. The resulting annealing peaks have been used for estimating the low temperature limit of $\beta$-relaxation, i.e., the temperature below which amorphous drugs would remain practically stable. A shift in the peak temperature with the heating rate allows one to obtain a trustworthy estimate for the activation energy of $\beta$-relaxation. The trustworthiness of the estimates has been tested by comparing them against independent estimates obtained by DMA, TSDC as well as by relationship $E_{\beta}=(24 \pm 3) R T_{g}$. More than satisfactory agreement has been observed for all studied systems.

5. The local and global mobility, in two popular amorphous pharmaceutical excipients, sucrose and trehalose, have been compared.

\section{Acknowledgment}

Thanks are due to Prof. S. Vyazovkin (University of Alabama) for help with DSC and DMA measurements.

\section{References}

[1]. Pouton, C. W. Eur. J. Polym.Sci., 2006, 29, 278-287.

[2]. Berstein, V.A.; Egorov, V.M. Differential ScanningCalorimetry of Polymers, Ellis Horwood, New York, 1994.

[3]. Brown, M.E. Introduction to Thermal Analysis, Techniques and Applications, Second Edition, 2001, Published by Kluwer Academic Publishers, 264 p.

[4]. Hohne, G.; Heminger, W.; Flammersheim, H.J. Differential Scanning Calorimetry "Springer-Velag, Berlin, 1999.

[5]. Menard, K.P.Dynamic Mechanical Analysis - A Practical Introduction, CRC Press, Boca Raton, USA, 1999.

[6]. Berstein, V. A.; Egorov, V.M. Polym. Sci. USSR, 27, 1985, 2743-2757.

[7]. Martin, A.; Swarbrick, J.; Cammarata, A. Physical Pharmacy, Lea and Febiger, Philadelphia, 1983

[8]. Hancock, C.; Shamblin, S.L. Zografi, G. Pharm.Res., 1995, vol. 12, No. 6, 799-806.

[9]. J. Swarbrick and J. Boylan, Encyclpedia of pharmaceutical technology, $2^{\text {nd }}$ Edition, 2002, Vol. $2,1132$.

[10]. Jackson, K.; Young, D. Pant, S. Pharmaceutical Science and Technology today, 2000, 3, 10, 336-345.

[11]. Wels, J. Pharmaceutical Preformulation, the physicochemical properties of drug substances, 1988.

[12]. Kudlik, A. et al. J. Mol. Struct., (1999) 479: 201-218. 